\title{
Acute Kidney Injury in Children: Look for It and Don't Ignore It!
}

\author{
Anil Vasudevan And Kishore D Phadke \\ Department of Pediatric Nephrology, Children's Kidney Care Centre, St John's Medical College Hospital, Bangalore, India. \\ kishorephadke@yahoo.co.in
}

A cute kidney injury (AKI), formerly known as acute renal failure (ARF) is an important contributor to mortality and morbidity, especially in critically ill children. Understanding the burden of AKI and its implication has been hampered by the lack of a precise definition of AKI. More than 30 definitions in literature have led to reporting of wide variation in incidence and outcomes [1]. A minor acute reduction in kidney function (which may be clinically not apparent and may be just a subtle rise in serum creatinine) has been consistently shown to have an adverse prognosis. Hence the term acute kidney injury (AKI) was proposed to encompass the entire spectrum not limited to those who need renal replacement therapy (RRT). Two similar definitions based on creatinine and urine output for diagnosis and classification of AKI; the pediatric Risk, Injury, Failure, Loss, End-Stage Renal Disease criteria (pRIFLE) and the Acute Kidney Injury Network (AKIN) staging system have been proposed [2,3].

The spectrum and burden of AKI in developing countries may be different from that of developed countries. The patients from developing countries are younger, infection associated AKI is more common and a significant proportion may have already developed AKI at the time of hospitalization. In addition, resource limitations in managing children who require RRT add to the burden.

Mehta and colleagues must be congratulated for taking the first step toward understanding the natural history of AKI in hospitalized children in our country using the AKIN criteria [4]. They observed that one third of critically ill children developed AKI in hospital and the incidence was four fold higher compared to the noncritically ill. The authors have validated the findings from world literature that AKI is common. They have shown that AKI occurs very early in hospitalization, which may have important bearing on future studies for early detection and treatment. AKI was associated with longer stay in hospital and four fold increase in mortality as compared to those who did not develop AKI reinforcing the importance of AKI as one of the important factor affecting morbidity and mortality. Not surprisingly, young age and sicker children were at increased risk of AKI. Nearly half of survivors with AKI did not have complete recovery, highlighting the importance of following up these children after discharge.

The authors have stated that most common cause of AKI is acute tubular necrosis (ATN). This may be an exaggeration as ATN is a histopathological diagnosis and many of the conditions leading to AKI may only affect glomerular filtration without compromising tubular integrity. As seen in the study, one of the major limitations of AKIN criteria is that children who do not have a baseline creatinine or who have AKI at admission in whom serum creatinine is already elevated would be missed. This will result in underestimating the incidence of AKI in a country like ours where a significant proportion may have already developed AKI at the time of hospitalization. Hence it would have been valuable to evaluate $p$ RIFLE in comparison to AKIN staging criteria and their influence on outcome and risk factors for AKI. It is also known that both the criteria identify somewhat different patients, particularly in small children in whom small changes may have large effect on AKI classification [5]. In order to overcome the lack of baseline creatinine, it would have been interesting to use baseline creatinine estimated by formula or use of age and gender specific norms and compare it with measured baseline serum creatinine [6].

This study has shown that AKI is common and harmful. As rightly pointed out by the authors, the study is underpowered to examine the effect of AKI as an independent risk factor for mortality. Adequately powered multicentre studies are required to generalize the observations from the current study and also comprehensively identify "at risk" population and evaluate short and long term outcomes. These studies would also lay the foundation for evaluating more robust urine and serum bio-markers in early identification and prognostication of AKI, given the limitations of serum creatinine as a biomarker. The initiation of nationwide AKI registry by Indian Society of Pediatric Nephrology to capture the spectrum of AKI is a welcome step [7].

Competing interests: None stated. Funding: Nil

\section{REFERENCES}

1. Bellomo R, Ronco C, Kellum JA, Mehta RL, Palevsky P; Acute Dialysis Quality Initiative workgroup. Acute renal failure: Definition, outcome measures, animal models, 
fluid therapy and information technology needs: The Second International Consensus Conference of the Acute Dialysis Quality Initiative (ADQI) Group. Crit Care. 2004;8:R204-12.

2. Acute Kidney Injury Network. Mehta RL, Kellum JA, Shah SV, Molitoris BA, Ronco C, Warnock DG, et al. Acute Kidney Injury Network: Report of an initiative to improve outcomes in acute kidney injury. Crit Care. 2007;11:R31.

3. Akcan-Arikan A, Zappitelli M, Loftis LL, Washburn KK, Jefferson LS, Goldstein SL. Modified RIFLE criteria in critically ill children with acute kidney injury. Kidney Int. 2007;71:1028-35.

4. Mehta P, Sinha A, Sami A, Hari P, Kalaivani M, Gulati A, et al. Incidence of acute kidney injury in hospitalized children. Indian Pediatr. 2012;49:537-42.

5. Joannidis M, Metnitz B, Bauer P, Schusterschitz N, Moreno $\mathrm{R}$, Druml $\mathrm{W}$, et al. Acute kidney injury in critically ill patients classified by AKIN versus RIFLE using the SAPS 3 database. Intensive Care Med. 2009;35:1692-1702.

6. Alkandari O, Eddington KA, Hyder A, Gauvin F, Ducruet $\mathrm{T}$, Gottesman $\mathrm{R}$, et al. Acute kidney injury is an independent risk factor for pediatric intensive care unit mortality, longer length of stay and prolonged mechanical ventilation in critically ill children: a two-center retrospective cohort study. Crit Care. 2011; 15:R146.

7. Indian Society of Pediatric Nephrology. AKI Registry Available from URL: http://124.247.216.178:8080/AKI. Accessed April 17, 2012. 